\title{
Numerical investigation of stiffness and buckling response of simple and optimized infill structures
}

\author{
Wang, Fengwen; Sigmund, Ole
}

Published in:

Structural and Multidisciplinary Optimization

Link to article, DOI:

$10.1007 / \mathrm{s} 00158-020-02525-3$

Publication date:

2020

Document Version

Peer reviewed version

Link back to DTU Orbit

Citation (APA):

Wang, F., \& Sigmund, O. (2020). Numerical investigation of stiffness and buckling response of simple and optimized infill structures. Structural and Multidisciplinary Optimization, 61, 2629-2639.

https://doi.org/10.1007/s00158-020-02525-3

\section{General rights}

Copyright and moral rights for the publications made accessible in the public portal are retained by the authors and/or other copyright owners and it is a condition of accessing publications that users recognise and abide by the legal requirements associated with these rights.

- Users may download and print one copy of any publication from the public portal for the purpose of private study or research.

- You may not further distribute the material or use it for any profit-making activity or commercial gain

- You may freely distribute the URL identifying the publication in the public portal

If you believe that this document breaches copyright please contact us providing details, and we will remove access to the work immediately and investigate your claim. 


\title{
Numerical investigation of stiffness and buckling response of simple and optimized infill structures
}

\author{
Fengwen Wang, Ole Sigmund
}

Received: date / Accepted: date

\begin{abstract}
This study systematically investigates stiffness and buckling response of finite structures consisting of repeated unit cells of a reference truss lattice microstructure and a topology optimized microstructure with enhanced buckling strength. Structural stability is evaluated using linear buckling, nonlinear pre-buckling, and post-buckling analyses, subjected to two benchmark loading cases representing uniaxial compression and shear loading. Numerical results indicate that geometric and material nonlinearities play a surprisingly small role in uniaxial loading, whereas strong effects are seen for the shear loading case for which the microstructure was not optimized.
\end{abstract}

Keywords Structural stability . Finite periodic structures · Linear buckling analysis - Nonlinear pre-buckling analysis · Post-buckling analysis

\section{Introduction}

Advances in additive manufacturing facilitate the fabrication of functional materials with unprecedented complexity $[1,2]$. This leads to increasing attention on architected materials exhibiting enhanced properties and multi-functionalities and in designing multi-level hierarchical lattice structures. Materials with exotic properties have for decades been systematically designed using topology optimization methods [3], covering extreme stiffness $[4,5,6,7]$, prescribed negative Pois-

Department of Mechanical Engineering

Technical University of Denmark

Nils Koppels Allé 404, 2800 Kongens Lyngby, Denmark

F. Wang

E-mail: fwan@mek.dtu.dk

O. Sigmund

E-mail: Sigmund@mek.dtu.dk son's ratio $[5,8,9,10]$, programmable auxetic behaviors $[11,12]$, zero and negative thermal expansion coefficients [13, 14], extreme buckling strength [15, 16] and acoustic topological insulators [17]. Recently, topology optimization methods have been employed to design structures with enhanced stiffness considering 3D printing infill $[18,19]$ or mapped first-level hierarchical materials $[20,21]$.

In addition to stiffness, buckling failure strength in materials or structures is also a fundamental and challenging issue. Even though structures with 3D printing infills in [19] or mapped first-level hierarchical materials in $[20,21]$ do not outperform their solid counterparts regarding stiffness, they may possess an advantage from the buckling strength perspective [22]. Material instabilities may develop with different wavelengths spanning from highly localized modes on the micro-scale to long-wavelength modes on the macroscale, and material and geometric nonlinearities further complicate the material instability studies. Previous numerical studies have resorted to simplified models which employ homogenization methods for separating scales [23] and Bloch-Floquet theory for detecting microscopic and macroscopic instabilities [24]. A general methodology for characterizing material strength due to bifurcation failure was proposed in [25]. Later this method was used to form analytic expressions for the failure surfaces of simple honeycomb microstructures [26]. A wide range of material configurations has been investigated in search for improved buckling strength, including 2D honeycombs [27], lattice structures [28] and thin-walled structures [29]. It has been discovered that geometric attributes play an essential role in the material buckling strength. Topology optimization of material strength was first studied in [15], where only cell-periodic buckling modes were taken into account. 
Later, this work was extended to cover both local and global modes via the Bloch-Floquet theory [30]. More recently, materials with enhanced buckling strength [16] have been systematically designed using topology optimization methods for different macro-level stress situations based on the homogenization theory, linear buckling (LB) analysis and Bloch-Floquet theory. It was shown that the optimized first-order hierarchical materials outperform their non-hierarchical counterparts at the cost of decreased stiffness.

It is an on-going discussion whether structures optimized under LB analysis will perform well in reality or under more realistic non-linear modelling assumptions. Also, it is not clear how a microstructure optimized under assumption of infinite periodicity will perform in a finite strain modelled, finite structure. Geometric and material nonlinearities as well as boundary effects may cause stiffening or softening effects depending on loading cases and microscale topologies. These open ends deserve further and more systematic investigations.

In this study, we aim at systematically investigating the performance of finite size structures formed from different periodic microstructures regarding stiffness and stability, based on different buckling strength evaluation criteria. The considered microstructures comprise a simple reference microstructure (RMS) and a topology optimized microstructure (OMS) with enhanced buckling strength from [16]. Two benchmark problems are formulated, i.e., uniaxial compression loading and shear loading. Although the OMS was optimized for hydrostatic loading, we consider the uni-axial loading case here in order to comply with on-going experimental verification studies. The buckling strength is evaluated using LB, nonlinear pre-buckling (NPB), and postbuckling (PB) analysis. The performance of structures with different infill periods is systematically investigated and compared. Numerical studies confirm the stability enhancement of the OMS in compression dominated finite structures. However, the linearly predicted indirect buckling enhancement in shear loading (not optimized for) degrades or even vanishes, due to nonlinear effects.

The remainder of the paper is organized as follows: Section 2 presents the benchmark problems, different approaches employed to evaluate structural buckling strength, and the hyperelastic material law describing the base material behavior under finite deformations. Section 3 presents the numerical results for the two benchmark problems. An extended discussion is provided in Section 4 and conclusions are drawn in Section 5.

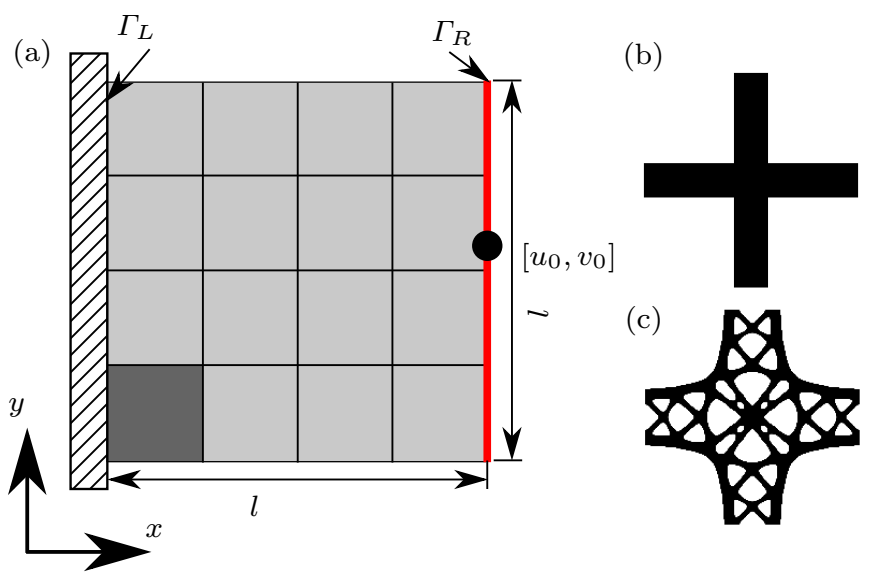

Fig. 1 (a) Schematic illustration of the benchmark problems for the considered structure consisting of $N$-by- $N$ unit cells with $l=10 \mathrm{~cm}$ and thickness of $t=1 \mathrm{~cm}$. The dark region represents one unit cell. $\Gamma_{L}$ and $\Gamma_{R}$ represent the left and right boundaries, respectively. (b) RMS. (c) First-order hierarchical OMS that optimizes buckling strength for hydrostatic compression (from [16]).

\section{Benchmark problems and computational methodologies for buckling evaluations}

In this section, we focus on presenting the benchmark problems considered in this study. Based on this, we present the computational approaches used to evaluate the structural buckling strength.

\subsection{Benchmark problems and considered infills}

In order to evaluate the performance of the considered material configurations, we consider a square structure infilled by $N$-by- $N$ unit cells with a constant size of $l=10 \mathrm{~cm}$ and thickness of $t=1 \mathrm{~cm}$. As illustrated in Fig. 1 (a), the left boundary of the structure is clamped, and the right is subjected to uniform displacements denoted by $\left[u_{0}, v_{0}\right]$. Under above boundary conditions, a uniaxial compression test can be mimicked by constantly reducing $u_{0}$ while keeping $v_{0}=0$. The zero vertical deflections at both ends are used to comply with physical compression tests where friction between loading surfaces and samples or an attached solid loading domain efficiently will work as fixed vertical boundary conditions. A shear loading test can similarly be mimicked by prescribing $v_{0}$ with free $u_{0}$.

Under above defined boundary conditions, the corresponding effective strain is

$$
\begin{array}{ll}
\varepsilon_{x x}=\frac{u_{0}}{l}, & \text { for uniaxial compression } \\
\varepsilon_{x y}=\frac{v_{0}}{2 l}, & \text { for shear loading }
\end{array}
$$


The considered infill microstructures are the reference microstructure (RMS) in Fig. 1 (b) and the topology optimized microstructure (OMS) with enhanced buckling strength for hydrostatic compression from [16] in Fig. 1 (c). Hereafter, we refer to them as the RMS and the OMS, unless otherwise stated. The first-order hierarchical configuration OMS is represented by the exact solution and discretization obtained in [16] but thresholded to a pure zero-one design where void elements are removed for simplification of analyses.

\subsection{Computational methodologies for buckling strength evaluations}

The finite element method is used to determine the critical buckling stress for the proposed benchmark structures. Three approaches are employed and investigated, i.e., LB, NPB, and PB analyses.

The LB analysis here assumes perfect structures, and hence geometric imperfections stemming from the manufacturing process are ignored. Under a small deformation assumption, the static equilibrium of the benchmark problem is governed by

$$
\begin{aligned}
\boldsymbol{K}_{0} \boldsymbol{u}_{0} & =\mathbf{0}, & & \\
\boldsymbol{u} & =\mathbf{0}, & & \text { on } \Gamma_{\mathrm{L}}, \\
\boldsymbol{u} & =\left[u_{0}, v_{0}\right]^{T}, & & \text { on } \Gamma_{\mathrm{R}} .
\end{aligned}
$$

Here $\boldsymbol{K}_{0}$ is the linear stiffness matrix and $\boldsymbol{u}_{0}$ is the equilibrium displacement vector. $\Gamma_{L}$ and $\Gamma_{R}$ denote the left and right boundaries, respectively. The total reaction forces on the right boundary are calculated using

$\boldsymbol{f}^{\mathrm{rect}}=\left[f_{x}^{\mathrm{rect}}, f_{y}^{\mathrm{rect}}\right]^{T}=\left[\sum_{i \in \Gamma_{R}} f_{x, i}^{\mathrm{int}}, \sum_{i \in \Gamma_{R}} f_{y, i}^{\mathrm{int}}\right]^{T}$.

Here $i$ refers to the nodal number and $f_{x, i}^{\text {int }}, f_{y, i}^{\text {int }}$ are the corresponding internal loads in the $x$ and $y$ direction, respectively,

Assuming that displacements at the buckling point are small and that stresses are proportional to the prescribed displacements on the boundaries, the stress stiffening effects due to mechanical loading can be evaluated in terms of the displacements determined by linear static analysis. The critical buckling stress is estimated by LB analysis via the following eigenvalue problem,

$\left[\boldsymbol{K}_{0}+\tau \boldsymbol{K}_{\sigma}\left(\boldsymbol{u}_{0}\right)\right] \boldsymbol{\phi}=\mathbf{0}$

where $\boldsymbol{K}_{\sigma}\left(\boldsymbol{u}_{0}\right)$ is the stress stiffness matrix obtained based on linear elasticity [31], and $\phi$ is the buckling mode for the eigenvalue $\tau$. The critical buckling stress factor is estimated by the smallest eigenvalue $\tau_{1}$,

$$
\begin{aligned}
& \sigma_{x x}^{\text {cri }}=\tau_{1} \bar{\sigma}_{x x}=\tau_{1} \frac{f_{x}^{\text {rect }}}{l t}, \quad \text { for uniaxial compression } \\
& \sigma_{x y}^{\text {cri }}=\tau_{1} \bar{\sigma}_{x y}=\tau_{1} \frac{f_{y}^{\text {rect }}}{l t}, \text { for shear loading }
\end{aligned}
$$

where $\bar{\sigma}_{x x}$ and $\bar{\sigma}_{x y}$ are the averaged stress in the $x$ direction and the averaged shear stress, respectively.

Alternatively, one can perform geometric nonlinear analysis on a perfect structure with repeated buckling analysis, to more accurately predict the buckling strength in the deformed state. Here, we use the total Lagrangian approach for the description of geometric nonlinearity. The structural equilibrium is controlled by

$\boldsymbol{r}(\boldsymbol{u})=f^{\text {int }}(\boldsymbol{u})=\mathbf{0}$.

Here $\boldsymbol{u}$ is the nodal displacement vector and $\boldsymbol{r}(\boldsymbol{u})$ and $\boldsymbol{f}^{\text {int }}(\boldsymbol{u})$ are the corresponding residual and internal load vectors, respectively. The internal nodal force vector is defined by

$\boldsymbol{f}^{\text {int }}(\boldsymbol{u})=\sum_{e} \boldsymbol{f}_{e}^{\text {int }}\left(\boldsymbol{u}_{e}\right)=\sum_{e} \frac{\partial\left(\int_{v_{e}} W\left(\boldsymbol{u}_{e}\right) d v\right)}{\partial \boldsymbol{u}_{e}}$.

Here $e$ represents the element number and $W\left(\boldsymbol{u}_{e}\right)$ is the stored elastic energy density defined by a hyperelastic material law. The corresponding tangent stiffness matrix is written as

$$
\boldsymbol{K}_{t}(\boldsymbol{u})=\sum_{e} \boldsymbol{K}_{t e}=\sum_{e} \frac{\partial \boldsymbol{f}_{e}^{\mathrm{int}}}{\partial \boldsymbol{u}_{e}}=\boldsymbol{K}(\boldsymbol{u})+\boldsymbol{K}_{\sigma}(\boldsymbol{u}),
$$

where $\boldsymbol{K}_{t e}$ is the elemental tangent stiffness of element, $e, \boldsymbol{K}(\boldsymbol{u})$ is the displacement dependent elastic stiffness matrix and $\boldsymbol{K}_{\sigma}(\boldsymbol{u})$ is the stress stiffness matrix stemming from the second Piola-Kirchhoff stress.

The static equilibrium, Eq. (6), is solved iteratively using the arc-length method in [32] via the incremental equation at the $n$th step stated as

$$
\begin{array}{cc}
\boldsymbol{K}_{t}\left(\boldsymbol{u}^{n}, \alpha^{n}\right) \Delta \boldsymbol{u}^{n}= & \boldsymbol{r}^{n}, \quad i \in \Gamma_{L}, \\
\boldsymbol{u}_{i}^{n}=\mathbf{0}, \quad & \\
\text { uniaxial compression : } & \\
\boldsymbol{u}_{x, i}^{n}=\alpha^{n} \Delta u_{0}, \boldsymbol{u}_{y, i}^{n}=0, & i \in \Gamma_{R}, \\
\text { shear loading : } & \\
\boldsymbol{u}_{y, i}^{n}=\alpha^{n} \Delta v_{0}, & i \in \Gamma_{R},
\end{array}
$$

Here $\Delta \boldsymbol{u}^{n}$ is the incremental displacement vector, $\alpha$ is the control parameter according to the applied boundary displacements. $\boldsymbol{u}_{x, i}^{n}$ and $\boldsymbol{u}_{y, i}^{n}$ are displacements of the node $i$ along the $x$ and $y$ direction, respectively, 
$\Delta u_{0}$ and $\Delta v_{0}$ are the prescribed displacement increments. Detailed description of the arc-length algorithm can be found in [32].

At each equilibrium state, an upcoming buckling point may be predicted based on the current tangent stiffness. Assuming the current configuration is close to the critical point, the stress stiffness part of the tangent stiffness at the critical point is approximated by extrapolating the nonlinear stress stiffness from the current configuration using a factor $\gamma$, while the elastic stiffness is assumed to not change with additional loading [31]. Hence, the critical factor can be approximated by an eigenvalue problem as

$\left[\boldsymbol{K}(\boldsymbol{u})+\gamma \boldsymbol{K}_{\sigma}(\boldsymbol{u})\right] \boldsymbol{\phi}=\mathbf{0}$.

The critical buckling stress is determined by the smallest eigenvalue of Eq. (10) and is written as

$\sigma_{x j}^{\text {cri }}=\gamma_{1} \bar{\sigma}_{x j}=\gamma_{1} \frac{f_{j}^{\text {rect }}}{l t}, \quad j \in\{x, y\}$

where the definition of $f_{j}^{\text {rect }}$ is the same as in Eq. (3) for different loading cases. Different from the linear case, the internal force is here calculated based on finite deformations using Eq. (7). It is remarked that $\gamma_{1}>1$ indicates that the current deformation is below the buckling point and is stable, and $\gamma_{1}<1$ indicates that the structure is beyond the buckling point. $\gamma_{1}=1$ signifies the stability limit. Hence the intersection between the critical buckling prediction and the stress-strain curve is the critical buckling stress. This procedure is called NPB analysis in this study.

In $\mathrm{PB}$ analysis, the same nonlinear analysis is performed using the arc-length method, however, with the presence of geometric imperfections. Geometric imperfections stemming from the manufacturing procedure stimulate buckling in the nonlinear analysis. The critical buckling stress can be detected by monitoring the tangent stiffness defined by

$\bar{E}_{x j}=\frac{\Delta \sigma_{x j}}{\Delta \varepsilon_{x j}}, \quad j \in\{x, y\}$

The buckling point in the PB analysis is defined by the state where the structure looses considerable stiffness for the first time. Here we define this state as the load where the stiffness has decreased to $20 \%$ of the initial one.

The geometric imperfections here are represented by a set of linear buckling modes with maximum perturbation amplitude, $\varphi$. The geometric imperfections are taken into account by modifying each nodal location according to the considered buckling modes scaled by $\varphi /|\max (\phi)|$. Nodal positions are equal to the sum of the original positions and perturbations from the buckling modes.

\subsection{Hyperelastic material law}

We utilize a modified compressible Neo-Hookean material law [33] to describe the material behavior under finite deformations. The corresponding material stored energy density is written as

$W=\frac{1}{2} \lambda_{0}(J-1)^{2}+\frac{\mu_{0}}{2}(I-3)-\mu_{0} \ln (J)$,

where $\boldsymbol{F}$ is the deformation gradient and $J=\operatorname{det}(\boldsymbol{F})$; $I=\operatorname{trace}(\mathrm{C})$ with $\boldsymbol{C}=\boldsymbol{F}^{T} \boldsymbol{F}$ being the right CauchyGreen deformation tensor; $\lambda_{0}$ and $\mu_{0}$ are the Lamé parameters of the base material.

The benchmark problems are assumed to be under the plane stress assumption, and Young's modulus and Poisson's ratio of the base material are $E_{0}=1$ and $\nu=0.3$. This corresponds to the values used in [16]. When considering finite deformations, it is necessary to modify the constitutive terms to enforce zero stress in the out-of-plane direction and thus account for the deformation gradient in the out-of-plane direction. The detailed implementation follows Chapter 6.2.4 in [33] and is not stated here.

In the $\mathrm{PB}$ analysis, the convergence criterion is set to be $\|\boldsymbol{r}\|<10^{-4}\left\|\boldsymbol{r}_{0}\right\|$ with a maximum of 30 iterations for each displacement increment. Here $\boldsymbol{r}_{0}$ is the linear reaction force vector resulting only from the prescribed displacement increment. In the uniaxial compression case, the displacement increment for the OMS is $\Delta u_{0}=0.0280 \mathrm{~cm}$, and a smaller displacement increment of $\Delta u_{0}=0.0040 \mathrm{~cm}$ is used for the RMS since its buckling strain is much smaller compared to the OMS. In the shear loading case, the displacement increment of $\Delta v_{0}=0.200 \mathrm{~cm}$ is employed. The increment steps are set to be 50 for all the cases. If the arclength method does not converge within 30 iterations in one displacement increment step, the displacement increment is halved, and the displacement increment step restarts. If the arc-length method converges and displacement increment is smaller than the initial one, the displacement increment is doubled for the next displacement increment.

\section{Numerical results}

Structures consisting of 2-by-2, 4-by-4, 6-by-6, and 8by- 8 unit cells are considered to account for size effects while keeping the structural size fixed. Each unit cell is discretized by $200 \times 2004$-node quadrilateral finite elements as in [16]. The volume fraction of the unit cells presented in Fig. 1 (b) and (c) is $30 \%$, and void regions are eliminated in the numerical simulations. Both the microstructures are orthotropic and possess 
the same properties along the two axial directions due to square symmetry. First, we consider the infinitely periodic case. Tab. 1 presents the effective material properties of the two infinitely periodic materials and the corresponding material buckling strength under uniaxial compression $\left(\sigma_{x x}^{\mathrm{cri}}\right)$ and under pure shear loading $\left(\sigma_{x y}^{\text {cri }}\right)$. They are estimated by employing the homogenization method and Bloch-Floquet theory to capture both long- and short-wavelength buckling modes as presented in [16].

As seen in Tab. 1, the OMS exhibits smaller bulk and Young's moduli than the RMS, whereas it possesses much higher shear modulus. The material buckling strength of the OMS is 4.5 times the one of the RMS under uniaxial compression. Fig. 2 (a) and (b) display the critical material buckling modes under uniaxial compression extrapolated over 4-by-4 unit cells. The critical buckling mode of the RMS is a global shear mode, whereas the one of the OMS is an anti-periodic mode spanning two cells. It is worthwhile mentioning that a cluster of material buckling modes in the OMS display buckling stress very close to the critical one.
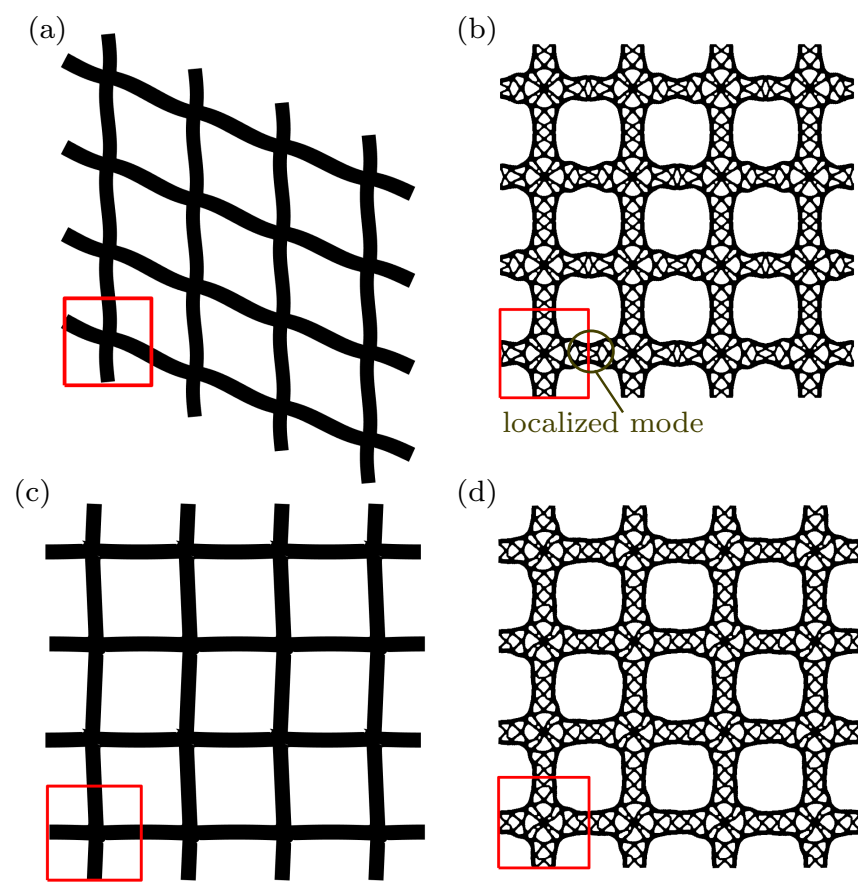

localized mode

(d)

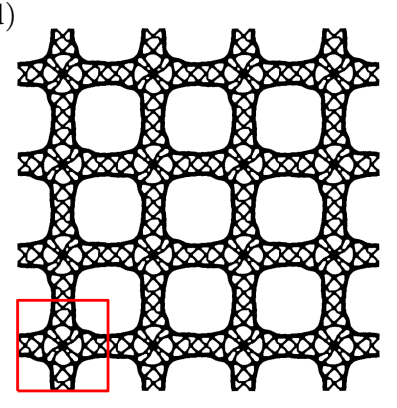

Fig. 2 Critical material buckling modes for infinitely periodic case, for the unixial compression (Top: (a) and (b)) and pure shear loading (Bottom: (c) and (d)).

Fig. 2 (c) and (d) present the critical material buckling modes for pure shear loading. The buckling mode in the RMS expands over two periods in the vertical direction, while the one in the OMS is an anti-periodic mode in both the $x$ - and $y$-directions. The buckling strength
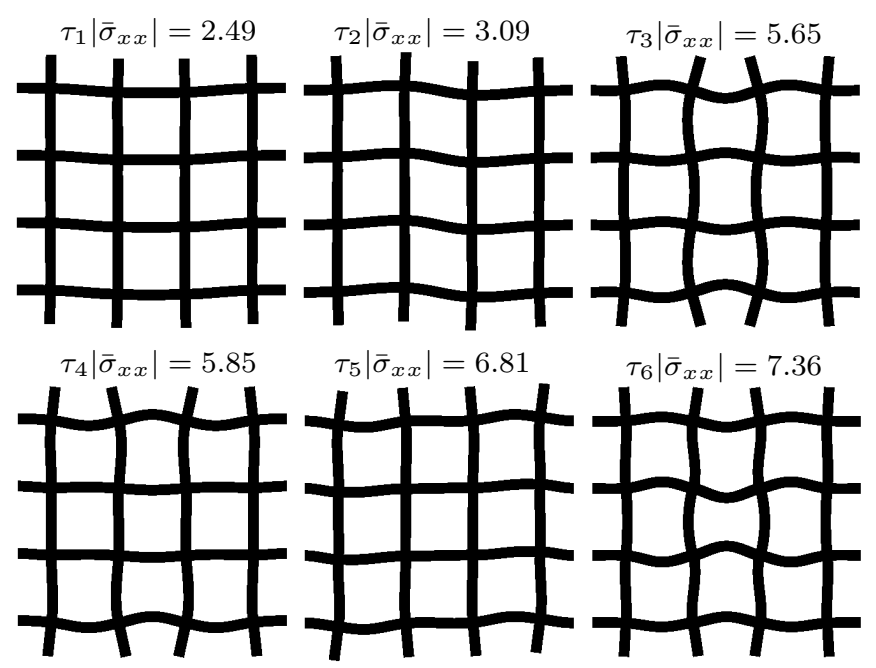

Fig. 3 First six buckling modes obtained from the LB analysis of the structure formed from the RMS with corresponding buckling stress $\left[10^{-3}\right]$. Top row from left to right: the first, second and third buckling modes; Bottom row from left to right: the forth, fifth and sixth buckling modes.

enhancement of the OMS degrades to be around 3.3 times that of the RMS.

In the linear finite cell analysis, the effective Young's modulus is measured from the uniaxial compression as $E^{H}=\sigma_{x x} / \varepsilon_{x x}$, and the effective shear modulus is measured from the shear loading as $G^{H}=\sigma_{x y} /\left(2 \varepsilon_{x y}\right)$. Tab. 2 summarizes the effective Young's and shear moduli measured for the structures with different periods. For the RMS, the measured properties converge to the effective material properties predicted using the homogenization method for infinite periodicity. This is not the case for the OMS, where Young's modulus deviates by $2 \%$ and shear modulus by $22 \%$. The main reason for this deviation is the difference in effective Poisson's ratio between the RMS with a near-zero Poisson's ratio and OMS with a finite Poisson's ratio. For zero Poisson's ratio, the fixed vertical displacement boundary conditions at left and right edges have no effect on the macroscopic response, whereas the opposite is the case when the Poisson's ratio is non-zero as in the OMS case.

\subsection{Buckling evaluation for uniaxial compression}

Under uniaxial compression, Fig. 3 shows the first six linear buckling modes of the structure formed from 4by- 4 periods of the RMS. It is seen that the critical buckling mode is the global shear mode in accordance with the critical material mode shown in Fig. 2. However, due to boundary stiffening effects, the structural critical buckling strength is around $12 \%$ higher than that for the infinitely periodic case. 
Table 1 Effective material properties for the infinitely periodic materials and material buckling stress for uniaxial compression and pure shear stress.

\begin{tabular}{lllllll}
\hline & $E^{H}$ & $\nu^{H}$ & $G^{H}$ & $\kappa^{H}$ & $\sigma_{x x}^{\text {cri }}\left[10^{-3}\right]$ & $\sigma_{x y}^{\text {cri }}\left[10^{-3}\right]$ \\
\hline RMS & 0.1638 & 0.0592 & 0.0024 & 0.0871 & 2.21 & 1.86 \\
OMS & 0.0952 & 0.3553 & 0.0162 & 0.0738 & 9.88 & 6.19 \\
\hline
\end{tabular}

Table 2 Measured Young's and shear moduli in finite structures

\begin{tabular}{lllllll}
\hline & infill & $N=2$ & $N=4$ & $N=6$ & $N=8$ & $N=\infty$ \\
\hline \multirow{2}{*}{$E^{H}$} & RMS & 0.1642 & 0.1640 & 0.1640 & 0.1639 & 0.1638 \\
& OMS & 0.0950 & 0.0960 & 0.0963 & 0.0963 & 0.0952 \\
\hline \multirow{2}{*}{$G^{H}$} & RMS & 0.0031 & 0.0025 & 0.0024 & 0.0024 & 0.0024 \\
& OMS & 0.0127 & 0.0128 & 0.0128 & 0.0127 & 0.0162 \\
\hline
\end{tabular}

In the PB evaluation, we first consider the imperfection stemming from the first buckling mode in Fig. 3 to investigate the influence of maximum perturbation amplitude on the PB evaluation considering the maximum perturbation amplitude of $\varphi=0.0001$ denoted by the army-green line and $\varphi=0.0005$ denoted by the black line in Fig. 4. In both cases, a small stiffening effect is observed, which leads to higher buckling stress in the PB analysis than the LB analysis represented by the blue line. The red circles in the lines highlight the critical buckling stress. The intersection between the strain-stress curve for the perfect structure denoted by the green dash-dot line and the corresponding prebuckling prediction denoted by the red dash-dot line indicates the buckling stress from the NPB prediction. It is seen that higher perturbation amplitude leads to a smaller buckling stress in the PB evaluation, and that the buckling stress is closer to the NPB prediction as the perturbation amplitude reduces. The final deformation patterns in both cases are same as the first buckling mode in Fig. 3. Considering that the perturbation of $\varphi=0.0001$ is very small, we fix the max perturbation amplitude to be $\varphi=0.0005$, hereafter, unless otherwise stated.

Fig. 5 summarizes the PB evaluations for two different geometric imperfections stemming from buckling modes in Fig. 3, i.e., 1) the critical buckling mode; 2) an equal-weighted superposition of the first six modes. The structure with only the first imperfection performs slightly softer than the one with multiple superimposed imperfections under finite deformations. Hence, the first imperfection results in lower critical buckling strength. The deviation of the buckling strength in these two cases is small, however, and final deformation patterns are equal, i.e., the global shear mode (see Fig. 5 (b) and (c)). (a)

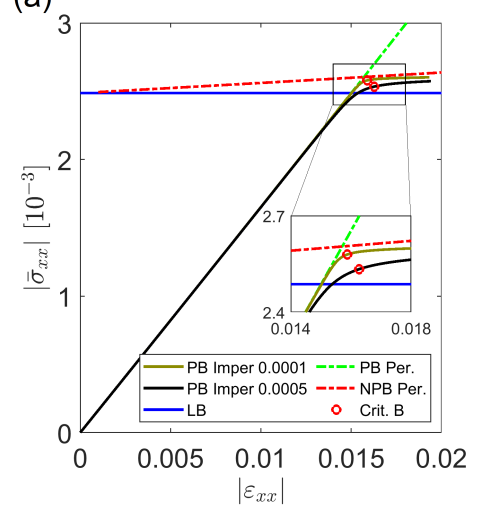

(b)

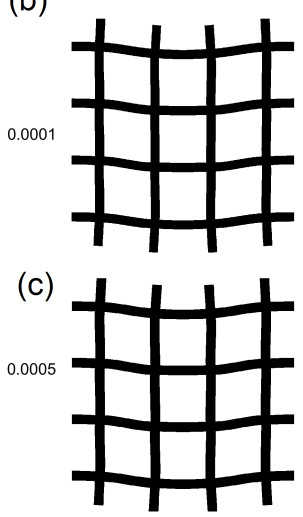

Fig. 4 Influence of imperfection amplitudes in the PB analysis. (a) Evaluation results. Structural deformation pattern for $\Phi=0.0001$ (b) and $\Phi=0.0005$ (c).
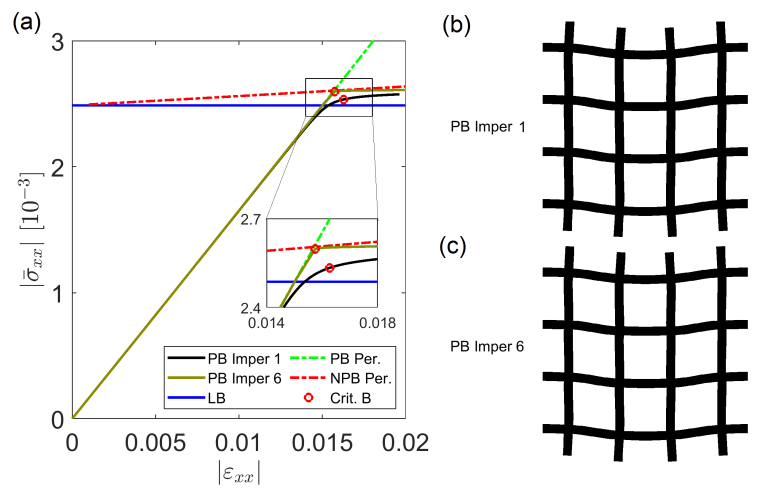

Fig. 5 (a) Stress-strain curves under uniaxial compression for the benchmark problem considering different imperfections. The red symbols indicate the critical buckling stress. (b) Deformation pattern with imperfection stemming from the first buckling mode; (c) Deformation pattern with imperfection stemming from the equal-weighted superposition of the first six buckling modes.

Next, we perform the same analysis for the optimized infill structure to investigate the influence of imperfection patterns on the $\mathrm{PB}$ evaluations. In contrast to the RMS structure, the first six buckling modes are all local modes located at interfaces between unit cells with close valued buckling stresses, as seen in Fig. 6 . This observation coincides with the material buckling analysis where a cluster of buckling modes exhibits similar buckling stresses. Moreover, the two different geometric imperfections result in two different buckling 
$\tau_{1}\left|\bar{\sigma}_{x x}\right|=9.33$

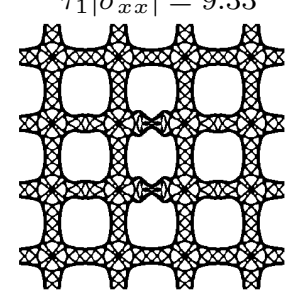

$\tau_{4}\left|\bar{\sigma}_{x x}\right|=9.40$
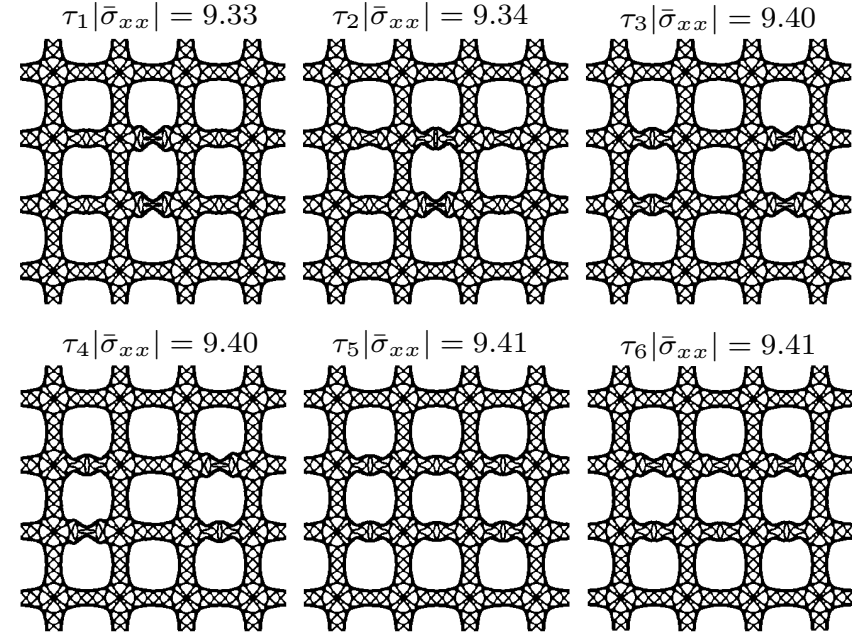

Fig. 6 First six buckling modes from LB analysis of the structure formed from the OMS with corresponding buckling stress $\left[10^{-3}\right]$. Top row: from left to right: the first, second and third buckling mode; Bottom row from left to right: the forth, fifth and sixth buckling modes.

modes in the PB evaluation, as shown in Fig. 7 (b) and (c). Structural softening is observed for both perturbed structures as illustrated by the reduced tangent stiffness in the stress-strain curve. Same as for the previous case, the second geometric imperfection leads to higher buckling stress in the PB evaluations. (a)

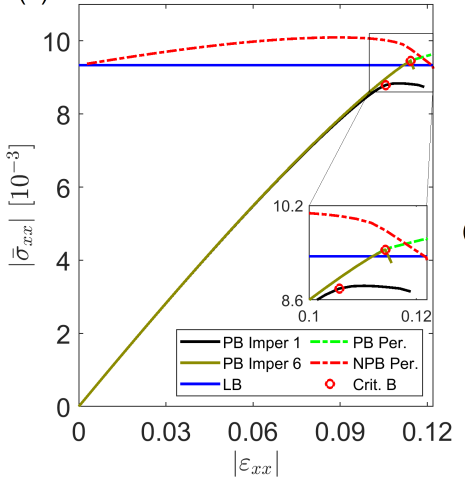

(b)

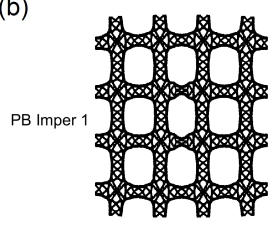

(c)

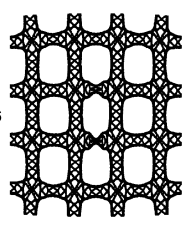

Fig. 7 (a) Stress-strain curves under the uniaxial compression of the structure infilled with the OMS considering different imperfections. The red symbols indicate the critical buckling stress; (b) Deformation pattern when imperfection stems from the first buckling mode; (c) Deformation pattern when imperfection stems from the equal-weighted superposition of the first six buckling modes.

Based on above observations, we focus on numerical investigations considering the geometric imperfection from the first buckling mode in the subsequent cases. Fig. 8 summarizes the critical buckling stress for both structures, obtained from the LB, PB and NPB analyses. It is seen that the OMS structure can undergo much larger deformations than the RMS and that it behaves softer and buckles at a higher stress level. For both structures, the buckling stress predicted from the NPB analysis is higher than the one from the LB analysis due to geometric and material nonlinearities, and geometric imperfections result in lower buckling stress compared to the one predicted from the NPB analysis. However, the difference between these two predictions is smaller for the RMS compared to the OMS. This observation further demonstrates that the OMS is more vulnerable to imperfections.

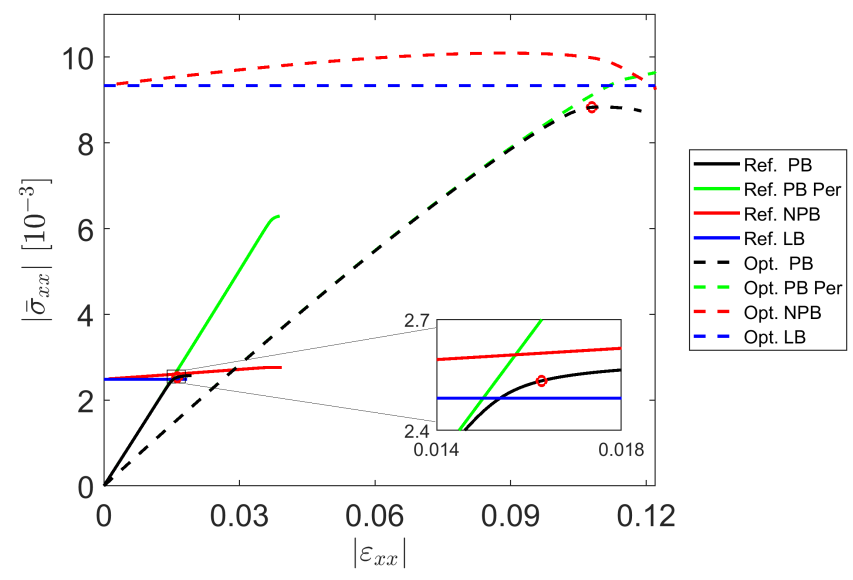

Fig. 8 Overall evaluations of the buckling stresses of structures formed from the RMS and OMS using the LB, PB and NPB analyses.

Fig. 9 shows the critical LB modes for structures with different numbers of infill periods and the corresponding buckling stresses. The critical buckling mode of the RMS structures is a global shear mode. The corresponding critical buckling stress decreases as the unit cell number increases and boundary stiffening effects diminish. However, for the OMS, the critical buckling modes are local modes 'dependent on the number of periods. The buckling modes are located at the two center unit cells in the vertical direction, but buckling modes span different unit cells in the lateral directions. The critical buckling stress stays almost constant as the unit cell period reaches 4 , and much smaller deviations are observed in the LB predicted buckling stress, compared to the RMS.

Tab. 3 summarizes the results for the structures with different periods of unit cells. It is seen that predicted buckling stresses using LB, PB and NPB analyses decrease as the unit cell period increases for the RMS. A dramatic decrease in the buckling stress is observed from 2-by-2 unit cells to more periods because the boundary stiffening effects are smaller as the periods increase. In general, slightly higher buckling stress 


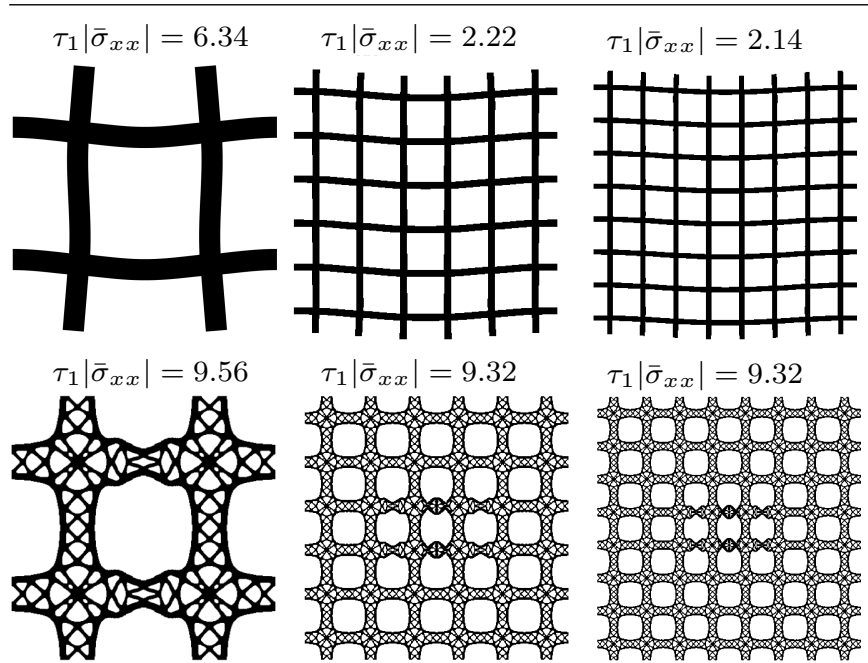

Fig. 9 First buckling modes of the structures with different periods with critical buckling stress $\left[10^{-3}\right]$. Top: RMS; Bottom: OMS

is obtained from the $\mathrm{PB}$ analysis than the one from the LB analysis due to stress stiffening effects. Moreover, the NPB analysis predicts the highest critical buckling stress due to geometric and material nonlinearities and lack of imperfections.

The overall assessment shows that the OMS structures exhibit much larger buckling stress than the RMS structures, which is in line with the linear material prediction. Hence, it may be concluded that optimization based on linear buckling analysis in this case has not overestimated the actual non-linear buckling response as otherwise often feared.

Table 3 Buckling strength of the structures with different periods of unit cells under the unixial compression evaluated using different numerical approaches $\left[10^{-3}\right]$.

\begin{tabular}{|l|lll|lll|}
\hline Unit cells & \multicolumn{3}{|c|}{ RMS } & \multicolumn{3}{c|}{ OMS } \\
\hline & LB & PB & NPB & LB & PB & NPB \\
\hline $\mathrm{N}=2$ & 6.34 & 6.86 & 7.10 & 9.56 & 9.48 & 9.56 \\
\hline $\mathrm{N}=4$ & 2.49 & 2.53 & 2.60 & 9.33 & 8.79 & 9.77 \\
\hline $\mathrm{N}=6$ & 2.22 & 2.24 & 2.31 & 9.32 & 9.21 & 9.76 \\
\hline $\mathrm{N}=8$ & 2.14 & 2.15 & 2.21 & 9.32 & 8.73 & 9.65 \\
\hline $\mathrm{N}=\infty$ & 2.21 & - & - & 9.88 & - & - \\
\hline
\end{tabular}

\subsection{Buckling evaluation for shear loading}

Next, we perform a similar analysis for shear loading keeping in mind that the OMS structure was not specifically optimized for shear loading.

Fig. 10 shows the critical buckling modes for the structures formed from 4-by-4 RMSs and OMSs. The critical buckling mode is a global shear deformation pattern for the RMS structure. The corresponding structural buckling stress is smaller than the material buckling stress listed in Tab. 1. Only a local boundary deformation pattern is observed in the OMS structure, where apparently, the critical buckling mode is induced by boundary effects, which cause stress concentrations at the lower-left corner. Hence, the corresponding structural buckling stress is much smaller than the material buckling stress. The OMS structure exhibits 2.4 times the buckling strength of the RMS structure in LB analysis. Compared to the material evaluation in Tab. 1, the buckling strength enhancement in the OMS structure degrades due to the boundary effects of the finite structures.
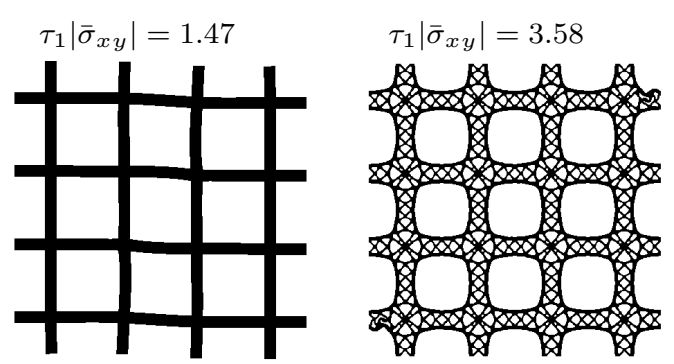

Fig. 10 Critical buckling modes under shear loading from LB analysis $\left[10^{-3}\right]$. Left: RMS. Right: OMS

Fig. 11 (a) summarizes the overall evaluations from the LB, NPB and PB analyses. The geometric imperfections used are the same as in the uniaxial compression case. Hence, in the PB analysis, the considered imperfections are fixed to be the first buckling modes shown in Fig. 10 with the maximum perturbation amplitude $\varphi=0.0005$.

Under shear loading, the OMS structure represented by dashed lines is stiffer than the RMS structure represented by solid lines. Deformation stiffening is observed in both structures due to the geometrically nonlinear stretching under shear loading. For the structure with the OMS, the PB analysis stops just below the NPB predicted buckling stress. For the structure with the RMS, no NPB prediction is available because no intersection between the strain-stress curve and pre-buckling prediction is observed. In the PB analysis, the arclength method is unable to find a converged solution using the employed computational strategy mentioned at the end of Section 2 after a certain number of increment steps. Hence the last converged result in the $\mathrm{PB}$ analysis is used as the buckling prediction from the PB analysis. The corresponding deformation patterns in Fig. 11 (b) and (c) demonstrate that both structures have buckled. Compared to the RMS structure, the OMS structure buckles at a much smaller shear 
strain. The corresponding buckling strength enhancement of the OMS is much smaller, compared to the results from the LB analysis and material prediction in Tab. 1 .

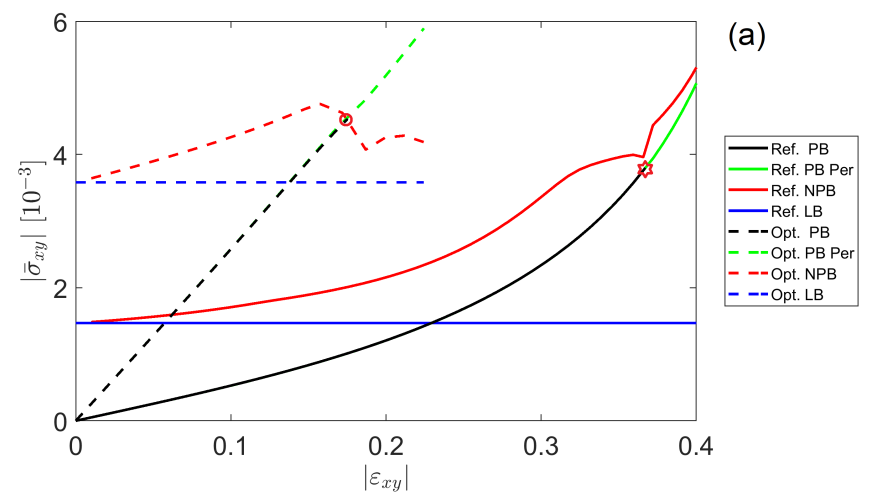

(b)

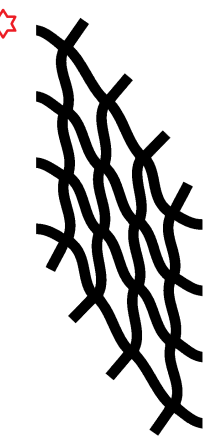

(c)

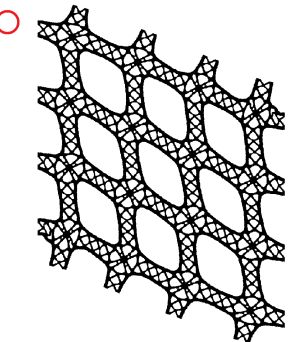

Fig. 11 (a) Overall evaluations of the buckling stress for the structures with the RMS and OMS using the LB, NPB and PB analyses under shear loading. (b) Deformation pattern of the structure with the RMS. (c) Deformation pattern of the structure with the OMS .

Tab. 4 summarizes the critical buckling stresses based on the LB and PB analyses. The LB predictions show that the buckling enhancement of the OMS structure degrades under shear loading. In the PB analysis, the last converged stresses are used to represent the critical buckling stress. It is seen that all the structures exhibit much higher buckling stresses in the PB analysis than the ones from the LB analysis, due to the geometrically nonlinear stretching under shear loading. Moreover, the critical buckling stress decreases as the period number increases. In contrast, the RMS structures show much higher buckling stress in the $\mathrm{PB}$ analysis than the material prediction. However, the OMS structures exhibit much lower buckling stress than the material prediction due to the boundary-induced buckling modes in the finite structures. Clearly, the predicted buckling strength from the material analysis has diminished due to boundary effects, material nonlinearities and geometrical non-linearities in particular.
Table 4 Buckling strength of the structures under shear loading with different periods of unit cells evaluated using different numerical approaches $\left[10^{-3}\right]$.

\begin{tabular}{|l|ll|ll|}
\hline Unit cells & \multicolumn{2}{|c|}{ RMS } & \multicolumn{2}{c|}{ OMS } \\
\hline & LB & PB & LB & PB \\
\hline $\mathrm{N}=2$ & 1.57 & 3.76 & 3.52 & 4.67 \\
\hline $\mathrm{N}=4$ & 1.47 & 3.88 & 3.58 & 4.46 \\
\hline $\mathrm{N}=6$ & 1.59 & 3.54 & 3.17 & 3.54 \\
\hline $\mathrm{N}=8$ & 1.63 & 2.95 & 2.86 & 3.19 \\
\hline $\mathrm{N}=\infty$ & 1.86 & - & 6.19 & - \\
\hline
\end{tabular}

\section{Discussion}

Our study confirms that finite structures infilled with OMSs optimized for infinite periodicity, infinitesimal strain assumption and linear buckling analysis in [16] can effectively enhance infill buckling strength with small decrease in stiffness, as illustrated by the uniaxial compression case. Hence, even if LB analysis does not take geometric and material nonlinear effects into account, it can effectively be used in a topology optimization process to produce efficient infill structures. However, our study also shows that LB predictions of shear buckling response, for which the OMS was not optimized, is highly inaccurate and cannot be trusted. This is not an unexpected conclusion since a softer structure in shear yields higher deformations that in turn cannot be predicted accurately by the linear model. Despite this conclusion, we note that the finite structures based on OMS still perform better in shear than the corresponding RMS-based structures.

We remark that an isotropic buckling optimized hierarchical triangular microstructure was also designed in [16]. It may in future studies be interesting to investigate if linear predictions of this microstructure subjected to shear loads are more accurate than the predictions for the orthotopic structure considered here. When only considering stiffness criteria, orthotropic infill is much more efficient than isotropic counterparts (c.f. [34]), however, more work still needs to be done to fully understand the connection between microscopic and macroscopic buckling criteria.

Another direction for future research is the use of finite deformations directly in the microstructure optimization formulation, possibly according to theory presented in $[24,25]$. Deeper investigations of geometrically non-linear effects in infinitely periodic structures are performed in our parallel work [35].

\section{Conclusion}

This study has systematically investigated the performances of simple and optimized periodic infill struc- 
tures (OMS) in terms of finite scale stiffness and buckling. The buckling is evaluated by linear buckling, nonlinear pre-buckling, and post-buckling analyses. Under uniaxial compression, the simple infill structures perform relatively linearly and buckle at small deformations, This means that all three evaluation methods provide close predictions on the critical buckling stress and hence that linear buckling analysis was sufficient as a base for the optimization in [16]. In contrast, the buckling strength enhancement of the OMS predicted using linear material buckling analysis diminishes in the finite structure evaluation under shear loading, due to boundary-induced buckling modes and geometric and material nonlinearities. Nevertheless, the overall evaluations indicate that buckling enhancement is significant when aligning the OMS along the principle stress direction compared to their simple RMS counterparts at little decrease in stiffness, supporting the conclusions in $[16]$.

\section{Replication of Results}

All simulations are performed using an in-house MATLAB implementation. The detailed formulations, hyperelastic material law, corresponding references, and parameters used in the $\mathrm{PB}$ analysis are listed in the paper to allow replication of results. The considered microstructures can be obtained on reasonable request.

\section{Conflict of interest}

The authors declare that they have no conflict of interest.

Acknowledgements The authors acknowledge the support of the Villum Fonden through the Villum Investigator Project InnoTop. The authors would like to thank Konstantinos Poulios, Gore Lukas Bluhm, Federico Ferrari in the Department of Mechanical Engineering at Technical University of Denmark for valuable discussions

\section{References}

1. L. Meza, A. J. Zelhofer, N. Clarke, A. J. Mateos, D. Kochmann, and J. Greer. Resilient 3D hierarchical architected metamaterials. Proceedings of the National Academy of Sciences, 112(37):1150211507, 2015.

2. X. Zheng, W. Smith, J. Jackson, B. Moran, H. Cui, D. Chen, J. Ye, N. Fang, N. Rodriguez, and T. Weisgraber. Multiscale metallic metamaterials. Nature materials, 15(10):1100, 2016.
3. M. P. Bendsøe and O. Sigmund. Topology Optimization: Theory, Methods and Applications. Springer, Berlin, 2003.

4. O. Sigmund. Materials with prescribed constitutuve parameters: An inverse homogenization problem. International Journal of Solids and Structures, 31(17):2313-2329, 1994.

5. O. Sigmund. Tailoring materials with prescribed elastic properties. Mechanics of Materials, 20(4):351-368, 1995.

6. J. K. Guest and J. H. Prévost. Optimizing multifunctional materials: design of microstructures for maximized stiffness and fluid permeability. International Journal of Solids and Structures, 43(2223):7028-7047, 2006.

7. X. Huang, A. Radman, and Y. M. Xie. Topological design of microstructures of cellular materials for maximum bulk or shear modulus. Computational Materials Science, 50(6):1861-1870, 2011.

8. U. D. Larsen, O. Sigmund, and S. Bouwstra. Design and Fabrication of Compliant Micromechanisms and Structures with Negative Poisson's Ratio. Journal of microelectromechanical systems, 6(2):99-106, 1997.

9. E. Andreassen, B. Lazarov, and O. Sigmund. Design of manufacturable 3D extremal elastic microstructure. Mechanics of Materials, 69(1):1-10, 2014.

10. P. Vogiatzis, S. Chen, X. Wang, T. Li, and L. Wang. Topology optimization of multi-material negative poisson's ratio metamaterials using a reconciled level set method. Computer-Aided Design, 83:15$32,2017$.

11. A. Clausen, F. Wang, J. S. Jensen, O. Sigmund, and J. A. Lewis. Topology Optimized Architectures with Programmable Poisson's Ratio over Large Deformations. Advanced Materials, 27(37):5523-5527, 2015.

12. F. Wang. Systematic design of 3D auxetic lattice materials with programmable Poisson's ratio for finite strains. Journal of the Mechanics and Physics of Solids, 114:303-318, 2018.

13. O. Sigmund and S. Torquato. Design of Materials With Extreme Thermal Expansion Using a ThreePhase Topology Optimization Method. J Mech Phys Solids, 45(6):1037-1067, 1997.

14. A. Takezawa and M. Kobashi. Design methodology for porous composites with tunable thermal expansion produced by multi-material topology optimization and additive manufacturing. Composites Part B: Engineering, 131:21-29, 2017.

15. M. M. Neves, O. Sigmund, and M. Bendsøe. Topology optimization of periodic microstructures with a 
penalization of highly localized buckling modes. International Journal for Numerical Methods in Engineering, 54(6):809-834, 2002.

16. C. R. Thomsen, F. Wang, and O. Sigmund. Buckling strength topology optimization of $2 \mathrm{D}$ periodic materials based on linearized bifurcation analysis. Computer Methods in Applied Mechanics and Engineering, 339:115-136, 2018.

17. R. E. Christiansen, F. Wang, and O. Sigmund. Topological insulators by topology optimization. Physical Review Letters, 122(23):234502, 2019.

18. X. Wang, S. Xu, S. Zhou, W. Xu, M. Leary, P. Choong, M. Qian, M. Brandt, and Y. M. Xie. Topological design and additive manufacturing of porous metals for bone scaffolds and orthopaedic implants: A review. Biomaterials, 83:127-141, 2016.

19. J. Wu, N. Aage, R. Westermann, and O. Sigmund. Infill optimization for additive manufacturing - approaching bone-like porous structures. IEEE transactions on visualization and computer graphics, 24(2):1127-1140, 2017.

20. J. P. Groen and O. Sigmund. Homogenizationbased topology optimization for high-resolution manufacturable microstructures. International Journal for Numerical Methods in Engineering, 113(8):1148-1163, 2018.

21. G. Allaire, P. Geoffroy-Donders, and O. Pantz. Topology optimization of modulated and oriented periodic microstructures by the homogenization method. Computers \&5 Mathematics with Applications, 2018.

22. A. Clausen, N. Aage, and O. Sigmund. Exploiting additive manufacturing infill in topology optimization for improved buckling load. Engineering, 2(2):250-257, 2016.

23. J. Guedes and N. Kikuchi. Preprocessing and postprocessing for materials based on the homogenization method with adaptive finite element methods. Computer methods in applied mechanics and engineering, 83(2):143-198, 1990.

24. G. Geymonat, S. Müller, and N. Triantafyllidis. Homogenization of nonlinearly elastic materials, microscopic bifurcation and macroscopic loss of rankone convexity. Archive for rational mechanics and analysis, 122(3):231-290, 1993.

25. N. Triantafyllidis and W. C. Schnaidt. Comparison of microscopic and macroscopic instabilities in a class of two-dimensional periodic composites. Journal of the Mechanics and Physics of Solids, 41(9):1533-1565, 1993.

26. B. Haghpanah, J. Papadopoulos, D. Mousanezhad, H. Nayeb-Hashemi, and A. Vaziri. Buckling of reg- ular, chiral and hierarchical honeycombs under a general macroscopic stress state. Proceedings of the Royal Society A: Mathematical, Physical and Engineering Sciences, 470(2167), 2014. 20130856.

27. N. Triantafyllidis and M. W. Schraad. Onset of failure in aluminum honeycombs under general in-plane loading. Journal of the Mechanics and Physics of Solids, 46(6):1089-1124, 1998.

28. L. R. Meza, G. P. Phlipot, C. M. Portela, A. Maggi, L. C. Montemayor, A. Comella, D. M. Kochmann, and J. R. Greer. Reexamining the mechanical property space of three-dimensional lattice architectures. Acta Materialia, 140:424-432, 2017.

29. F. G. Rammerstorfer, D. H. Pahr, T. Daxner, and W. K. Vonach. Buckling in thin walled micro and meso structures of lightweight materials and material compounds. Computational Mechanics, 37(6):470-478, 2006.

30. M. M. Neves, O. Sigmund, and M. P. Bendsoe. Topology optimization of periodic microstructures with a buckling criteria. Vienna University of Technology, 2002.

31. P. Wriggers. Nonlinear finite element methods. Springer Berlin Heidelberg, 2008.

32. S. Krenk. Non-linear modeling and analysis of solids and structures. Cambridge University Press, 2009.

33. O. C. Zienkiewicz and R. L. Taylor. The finite element method for solid and structural mechanics. Elsevier Butterworth-Heinemann, Amsterdam; Boston, 2005.

34. J. P. Groen, J. Wu, and O. Sigmund. Homogenization-based stiffness optimization and projection of $2 \mathrm{D}$ coated structures with orthotropic infill. Computer Methods in Applied Mechanics and Engineering, 349: 722-742, 2019.

35. G. L. Bluhm, O. Sigmund, F. Wang, and K. Poulios. Nonlinear compressive stability of hyperelastic 2D lattices at finite volume fractions. Journal of the Mechanics and Physics of Solids, 137: 103851. 